\title{
Coexistence of Stable Limit Cycles in a Generalized Curie-Weiss Model with Dissipation
}

\author{
Luisa Andreis ${ }^{1}$ (D) Daniele Tovazzi ${ }^{2}$
}

Received: 14 November 2017 / Accepted: 27 July 2018 / Published online: 2 August 2018

(c) Springer Science+Business Media, LLC, part of Springer Nature 2018

\begin{abstract}
In this paper, we modify the Langevin dynamics associated to the generalized Curie-Weiss model by introducing noisy and dissipative evolution in the interaction potential. We show that, when a zero-mean Gaussian is taken as single-site distribution, the dynamics in the thermodynamic limit can be described by a finite set of ODEs. Depending on the form of the interaction function, the system can have several phase transitions at different critical temperatures. Because of the dissipation effect, not only the magnetization of the systems displays a self-sustained periodic behavior at sufficiently low temperature, but, in certain regimes, any (finite) number of stable limit cycles can exist. We explore some of these peculiarities with explicit examples.
\end{abstract}

Keywords Collective periodic behavior · Generalized Curie-Weiss model · Hopf bifurcation · Interacting diffusion · Liénard systems · Mean field models · Phase transitions

\section{Introduction}

Occurrence of rhythmic behaviors is one of the most interesting phenomena observed in complex systems coming from life science. Since it is natural to model such systems by means of large families of interacting units, one question is how periodic behaviors emerge at macroscopic level when the single units have no tendency to behave periodically. In this framework, we say that we observe a self-sustained periodic behavior if there is a phase in which the equation for the evolution of the macroscopic law has a stable periodic solution, without the action of any periodic force: examples of this phenomenon come from biology, ecology and socioeconomics $[4,15,24,25]$. This periodicity, even if often detectable by numerical simulations, it is a peculiarity of the thermodynamic limit and it is quite hard to formally prove it, due to the infinite dimensional nature of the problem.

Luisa Andreis

luisa.andreis@wias-berlin.de

Daniele Tovazzi

tovazzi@math.unipd.it

1 WIAS-Weierstrass Institute, Mohrenstr. 39, 10117 Berlin, Germany

2 Dipartimento di Matematica “T. Levi-Civita”, Universitá di Padova, via Trieste 63, 35121 Padova, Italy 
Some recent works $[10,16]$ investigate the minimal hypothesis needed to create selfsustained periodic behavior in mean field interacting particle systems. One key step is to consider interactions that favor cooperation among units of the same type, but the reversibility of the dynamics seems to be in contrast with the occurrence of periodic behavior $[2,16]$. Therefore, a number of different mechanisms perturbing classical reversible models have been proposed. For example, the role of noise appears to be crucial: it may induce periodicity in systems whose deterministic version do not admit periodic solutions, i.e. noise-induced periodicity $[10,20,23]$ or it enhances periodicity in dynamical systems already proved to have limit cycles, i.e. excitability by noise $[7,18]$. Moreover, the addition of disorder in the initial phase of each rotator for the Kuramoto and other active rotators models, see [1,6,16], gives origin to oscillating behavior of the stationary solutions. In multi-populated models, it is instead a suitable structure of the interaction network, with or without delays, that produces periodicity, see [8,12,22].

In this paper, we focus on the mechanism that induces self-sustained periodic behavior by introducing a dissipation term in the interaction energy. In [9], the authors consider a dynamical Curie-Weiss model where each particle has its own local field that undergoes a diffusive and dissipative dynamics. In this case, due to the mean field interaction and of the spin-flip dynamics, it is possible to completely analyze the limiting system: the authors show that the introduction of dissipation prevents the system from relaxing to a magnetized equilibrium and this gives rise to a periodic orbit via a Hopf bifurcation. The general approach to introduce dissipation in systems of interacting diffusions is presented in [7], together with the study of limit cycles in the dissipative counterpart of a model of cooperative behavior introduced by Dawson [11]. Starting from these works, a natural question is whether the add of a dissipative term in a cooperative interaction is a robust tool that is able to create stable periodic orbit in systems otherwise in equilibrium. In this view, we aim to define models showing additional features than the ones above, but still analytically tractable. We consider the class of generalized Curie-Weiss models, defined in [14] and further studied in [13], being a straightforward extension of the classical Curie-Weiss Gibbs measure. It replaces the quadratic interaction function by a more general one and the spin- $\frac{1}{2}$ single site distribution by a symmetric distribution on $\mathbb{R}$. We define a dissipated version of the Langevin dynamics for this model and we analytically study the case where the single site distribution of spins is given by a Gaussian distribution. This case is of particular interest because the limiting behavior is given by a simple Gaussian process, reducing the nonlinear problem to a three-dimensional one. Moreover, the dissipation originates a Liénard system, for which an extended literature on limit cycles is present. Thanks to these features, we confirm that the dissipative mechanism is able to create self-sustained periodic behavior. In particular, we prove that coexistence of more than one stable limit cycles is possible in systems of this type, that is an interesting extension of existing models with dissipation in which at most one attractive periodic solution is possible. The model considered in this paper seems to be more general and flexible, since we give sufficient conditions on the interaction function in order to have as many periodic orbit as wanted.

The paper is organized as follows: in Sect. 2 we define the model with general single site distribution at a microscopic and at a macroscopic level and we state the main results on the dynamics with the Gaussian single site distribution. We analyze the situation in which more than one stable periodic orbit coexist in the limiting dynamics and we give sufficient conditions on the interaction function for this to happen. We illustrate the many different regimes that may be induced by the appropriate tuning of the interaction function with the qualitative analysis of an explicit example that shows coexistence of stable limit cycles. In Sect. 3 we prove the main result on the existence of periodic solution to the macroscopic equa- 
tion in certain regimes, through the study of a planar dynamical system. In Sect. 4 we prove well-posedness of the macroscopic nonlinear equation and the link between microscopic and macroscopic equation via propagation of chaos.

\section{The Model and Main Results}

Let us consider a generalized Curie-Weiss model, see [13], that is a sequence of probability measures on $\mathbb{R}^{N}$, for $N=1,2, \ldots$, given by

$$
\mathbf{P}_{N, \beta}\left(d x_{1}, \ldots, d x_{N}\right)=\frac{1}{Z_{N}(\beta)} \exp \left(N \beta g\left(\sum_{i=1}^{N} \frac{x_{i}}{N}\right)\right) \prod_{i=1}^{N} \rho\left(x_{i}\right) d x_{i},
$$

where $\rho$ is the density function of a symmetric probability measure on $\mathbb{R}$ with full support representing the single-site distribution of a spin in absence of interaction, $g$ is the interaction function, $\beta$ is the inverse temperature of the model and $Z_{N}(\beta)$ is the normalizing constant. For each $N$ fixed, a Langevin dynamics associated to (1) is a diffusion process $X^{N}$ with values in $\mathbb{R}^{N}$ such that $\mathbf{P}_{N, \beta}$ is its unique invariant measure, i.e. $X^{N}$ is solution to the following system of SDE

$$
d X_{i}^{N}(t)=\frac{\beta}{2} g^{\prime}\left(\frac{\sum_{j=1}^{N} X_{j}^{N}(t)}{N}\right) d t+\frac{\rho^{\prime}\left(X_{i}^{N}(t)\right)}{2 \rho\left(X_{i}^{N}(t)\right)} d t+d B_{t}^{i},
$$

where $\left\{B^{i}\right\}_{i=1, \ldots, N}$ is a family of independent 1-dimensional Brownian motions. The dynamics in (2) represent an interacting particle system where each particle follows its own dynamics, given by the last two terms on the right-hand side, and it experiences an interaction which depends on the empirical mean of the system $m^{N}(t):=\frac{\sum_{j=1}^{N} X_{j}^{N}(t)}{N}$. By following the approach in [7,9], we aim to define a dynamical model of interacting diffusions where the interaction depends on a "perceived magnetization" rather than on $m^{N}(t)$. Indeed, we introduce the variables $\lambda_{i}^{N}$, for $i=1, \ldots, N$, that evolve as the magnetization of the system but they undergo a dissipative and diffusive evolution and we substitute them in (2), obtaining

$$
\left\{\begin{array}{l}
d X_{i}^{N}(t)=\frac{\beta}{2} g^{\prime}\left(\lambda_{i}^{N}(t)\right) d t+\frac{\rho^{\prime}\left(X_{i}^{N}(t)\right)}{2 \rho\left(X_{i}^{N}(t)\right)} d t+d B_{t}^{i, 1}, \\
d \lambda_{i}^{N}(t)=-\alpha \lambda_{i}^{N}(t) d t+D d B_{t}^{i, 2}+d m^{N}(t),
\end{array}\right.
$$

where $\left\{\left(B^{i, 1}, B^{i, 2}\right)\right\}_{i=1, \ldots, N}$ are independent Brownian motions. When $\alpha=D=0$ the "perceived magnetization" is equivalent to the total magnetization of the system and the evolution (3) is equivalent to (2). To guarantee well-posedness of (3) we impose the following technical assumptions.

Assumption 1 Let $\alpha, D \geq 0$ and $\beta>0$ and the functions $g$ and $\rho$ satisfy the following:

(i) $g: \mathbb{R} \rightarrow \mathbb{R}_{>0}$ is an even, $C^{2}(\mathbb{R})$ function, strictly increasing on $[0, \infty)$ with $g(0)=0$.

(ii) $g^{\prime}$ is uniformly Lipschitz continuous, i.e. there exists a finite constant $L \geq 0$ such that for all $x, y \in \mathbb{R}$

$$
\left|g^{\prime}(x)-g^{\prime}(y)\right| \leq L|x-y| .
$$


(iii) $\rho$ is the density function of a symmetric Borel probability measure on $\mathbb{R}$ with full support, absolutely continuous w.r.t. the Lebesgue measure, such that $\log (\rho(x)) \in C^{2}$ and there exists $K>0$ s.t. for all $x, y \in \mathbb{R}$

$$
(x-y)\left(\frac{\rho^{\prime}(x)}{\rho(x)}-\frac{\rho^{\prime}(y)}{\rho(y)}\right) \leq K(x-y)^{2} .
$$

(iv) There exists a symmetric, nonconstant, convex function $h$ on $\mathbb{R}$ such that

$$
\begin{aligned}
& g(x) \leq h(x) \text { for all } x \in \mathbb{R}, \\
& \qquad \int_{\mathbb{R}} e^{a h(x)} \rho(x) d x<\infty \text { for all } a>0 .
\end{aligned}
$$

These are technical assumptions that mainly come from the definition of the generalized Curie-Weiss model in [13] and from the classical theory of mean field models. Points (i) and (iv) ensure that, for all $\beta>0$ and for all $N \in \mathbb{N}$, (1) is well defined, while points (ii) and (iii) are used to ensure well-posedness of (3) and of its McKean-Vlasov limit that we introduce below. Under Assumption 1 the generalized Curie-Weiss model is well-defined and, since the interactions are of mean field type, we expect a propagation of chaos result to hold. Therefore, we define the nonlinear Markov process $(X, \lambda)$ on $\mathbb{R}^{2}$, that is solution of the following nonlinear SDE:

$$
\left\{\begin{array}{l}
d X_{t}=\frac{\beta}{2} g^{\prime}\left(\lambda_{t}\right) d t+\frac{\rho^{\prime}\left(X_{t}\right)}{2 \rho\left(X_{t}\right)} d t+d B_{t}^{1} \\
d \lambda_{t}=-\alpha \lambda_{t} d t+\left\langle\mu_{t}(d x, d l), \frac{\beta}{2} g^{\prime}(l)+\frac{\rho^{\prime}(x)}{2 \rho(x)}\right\rangle d t+D d B_{t}^{2} \\
\mu_{t}=\operatorname{Law}\left(X_{t}, \lambda_{t}\right),
\end{array}\right.
$$

where $B=\left(B^{1}, B^{2}\right)$ is a two dimensional Brownian motion and the notation $\langle\alpha(d x), f(x)\rangle$ stands for $\int_{U} f(x) \alpha(d x)$, for all measures $\alpha$ on $U$ and measurable functions $f: U \rightarrow \mathbb{R}$. Well-posedness of (5) and the property of propagation of chaos are proved, with a rather standard approach, in Sect. 4. Collective periodic behavior is a phenomenon which appears only in this mean field limit. Indeed, the finite dimensional and irreducible dynamics of the $N$ particles system forbid the existence of more than one stationary solution, while the nonlinear process may admit invariant sets of measures. These are lower dimensional manifolds in which the dynamics of (5) is proven to be time-periodic. Moreover it is stable, in the sense that it is the long time limit for trajectories starting from initial condition in a certain basin of attraction of it. For this reason, in the following we study the long-time behavior of solutions of (5) under certain additional assumptions.

\subsection{Existence of Limit Cycles in the Gaussian Dynamics}

We choose to focus on the Gaussian dynamics, i.e. we choose as single site distribution of spins the Normal distribution with mean zero and variance $\sigma^{2}$. This means that we add the following set of assumptions.

Assumption 2 Let $D=0, \sigma>0$ and

$$
\rho(x):=\frac{1}{\sqrt{2 \pi \sigma^{2}}} e^{-\frac{x^{2}}{2 \sigma^{2}}} .
$$


(i) There exists a symmetric, nonconstant, convex function $h$ on $\mathbb{R}$ such that

$$
\begin{aligned}
& g(x) \leq h(x) \text { for all } x \in \mathbb{R}, \\
& \qquad \int_{\mathbb{R}} e^{a h(x)} e^{-x^{2}} d x<\infty \text { for all } a>0 .
\end{aligned}
$$

(ii) Let the initial condition measure be of the form

$$
\mu_{0}(d x, d \lambda)=v_{0}(d x) \times \delta_{\lambda_{0}}(d \lambda),
$$

where $v_{0}$ is a square-integrable measure on $\mathbb{R}$ and $\delta_{\lambda_{0}}$ is a Dirac delta centered in $\lambda_{0} \in \mathbb{R}$. Under Assumptions 1 and 2, the nonlinear process $(X(t), \lambda(t))_{t \geq 0}$, is solution of the following nonlinear SDE:

$$
\left\{\begin{array}{l}
d X_{t}=\frac{\beta}{2} g^{\prime}\left(\lambda_{t}\right) d t-\frac{X_{t}}{2 \sigma^{2}} d t+d B_{t}, \\
\frac{d \lambda_{t}}{d t}=-\alpha \lambda_{t}+\frac{\beta}{2} g^{\prime}\left(\lambda_{t}\right)-\frac{m_{t}}{2 \sigma^{2}}, \\
\mu_{t}=\operatorname{Law}\left(X_{t}, \lambda_{t}\right) \text { and } m_{t}=\left\langle\mu_{t}(d x, d l), x\right\rangle,
\end{array}\right.
$$

for $\left\{B_{t}\right\}$ Brownian motion. Clearly $\mu_{t}$ preserves the product form $v_{t}(d x) \times \delta_{\lambda_{t}}(d \lambda)$ and the resulting process is such that, if we define $v_{t}=\operatorname{Var}\left[X_{t}\right]$, the equations describing the evolution of the quantities $\left\{\left(m_{t}, v_{t}, \lambda_{t}\right)\right\}_{t \geq 0}$ are closed. In particular, when $v_{0}$ is a Gaussian distribution, then $\left\{X_{t}\right\}_{t \geq 0}$ is a Gaussian process. In the following we study the stability properties and the long-time behavior of (7) in this regime, where the $\lambda$ component is completely deterministic.

Theorem 1 Fix $\sigma^{2}>0$ and $\alpha>0$, for every $\beta>0$ the process $\left(X_{t}, \lambda_{t}\right)$ described by (7) has one stationary distribution for all $\beta>0$ of the form $\nu^{*}(d x) \times \delta_{\lambda^{*}}(d \lambda)$ and it is given by the measure

$$
\mu_{(0,0)}^{*}(d x, d l):=\frac{1}{\sqrt{2 \pi \sigma^{2}}} e^{-\frac{x^{2}}{2 \sigma^{2}}} d x \times \delta_{0}(d l) .
$$

(i) There exists $\beta^{*}>0$ such that $\forall \beta \in\left(0, \beta^{*}\right) \mu_{(0,0)}^{*}$ is the unique stationary solution for (7) and it is globally stable. Indeed, for all $\mu_{0}(d x, d \lambda)=v_{0}(d x) \times \delta_{\lambda_{0}}(d \lambda)$, squareintegrable initial conditions with $\lambda_{0} \in \mathbb{R}$,

$$
\lim _{t \rightarrow \infty}\left\|\mu_{t}(\cdot)-\mu_{(0,0)}^{*}(\cdot)\right\|_{T V}=0
$$

(ii) If $g^{\prime \prime}(0)>0$, let $\beta_{H}:=\frac{2 \alpha+\frac{1}{\sigma^{2}}}{g^{\prime \prime}(0)}$, then $\beta_{H} \geq \beta^{*}$ and for $\beta>\beta_{H}$ there exists at least one closed curve $\gamma \in \mathbb{R}^{2}$ such that the set

$$
\Gamma(\gamma)=\left\{\mu_{(m, \lambda)}^{*}(d x, d l):=\frac{1}{\sqrt{2 \pi \sigma^{2}}} e^{-\frac{(x-m)^{2}}{2 \sigma^{2}}} d x \times \delta_{\lambda}(d l), \text { for all }(m, \lambda) \in \gamma\right\}
$$

is an invariant manifold for the dynamics (7), that is time-periodic on $\Gamma(\gamma)$. Moreover, $\Gamma(\gamma)$ is stable, in the sense that it exists an open set $U(\gamma) \in \mathbb{R}^{2}$ such that for all $\mu_{0}(d x, d \lambda)=\nu_{0}(d x) \times \delta_{\lambda_{0}}(d \lambda)$, square-integrable initial conditions with $\left(\left\langle\mu_{0}, x\right\rangle, \lambda_{0}\right) \in U(\gamma)$, it holds

$$
\lim _{t \rightarrow \infty} \inf _{(m, \lambda) \in \gamma}\left\|\mu_{t}(\cdot)-\mu_{(m, \lambda)}^{*}(\cdot)\right\|_{T V}=0 .
$$

(iii) If $g^{\prime \prime}(0)>0$ and there exists $C>0$ such that for all $x \in(C, \infty)$ the function $g^{\prime}(x)$ is concave, then there exists a $\beta_{U C} \geq \beta_{H}$ such that for all $\beta>\beta_{U C}$ there exists a unique closed curve $\gamma \in \mathbb{R}^{2}$ such that $\Gamma(\gamma)$ is invariant for the dynamics (7) and (9) holds with $U(\gamma)=\mathbb{R}^{2} \backslash\{(0,0)\}$. 
In Theorem 1 we describe different parameter regimes of the dynamics (7). The picture is clearly not exhaustive, but it already shows some interesting features of the process. Point (i) says that, at sufficiently high temperatures, there is a unique equilibrium for the process, which is the disordered situation. In Point (ii) we highlight the existence, for certain types of interaction function, of a Hopf bifurcation. Then, for sufficiently low temperatures we see the existence of a set of measures which is invariant under the dynamics, which is time periodic on this set. This periodic solution of the system (7) is stable, in the sense that it exists a certain domain of attraction in the set of initial conditions, such that solutions of (7) with these initial conditions approach the invariant manifold. Point (iii) proves that, for a certain class of interaction function, there exists a regime in which we have uniqueness of the periodic solution and this solution attracts all the trajectories starting with a deterministic $\lambda$, except for the stationary one $\mu_{(0,0)}^{*}$. In the following subsection we underline the existence of many other regimes and in particular we give sufficient conditions for the emergence of regimes with an arbitrary number of periodic solution.

\subsection{Coexistence of Stable Limit Cycles}

As pointed out in [9], collective periodic behavior can be observed in certain regimes when dissipation is introduced in a classical Curie-Weiss model. In that particular model, a Hopf bifurcation in a dynamical system identifies the transition from disorder to a phase in which a unique globally stable limit cycle is present. The model introduced here generalizes this scheme: the proof of Theorem 1 will show that the study of limit cycles depends (as in [9]) on the study of a planar dynamical system of Liénard type, see [5,19]. In point ii) of Theorem 1 we describe what happens when a Hopf bifurcation occurs in this system but, according to the form of the interaction function $g(x)$, the limiting dynamics may display a richer behavior. The most interesting phenomena are the appearance of limit cycles when the origin is still locally stable and the coexistence of more than one limit cycles. These orbits, in general, are created and destroyed through global bifurcations. Let us give a general idea of what may happen, depending on the interaction function $g$. As we prove in Sect. 3, the limit cycles of (7) depend on the following dynamical system of Liénard type:

$$
\left\{\begin{array}{l}
\dot{y}_{t}=-\frac{\alpha}{2 \sigma^{2}} \lambda_{t} \\
\dot{\lambda}_{t}=y_{t}-f_{\alpha, \beta}\left(\lambda_{t}\right)
\end{array}\right.
$$

with

$$
f_{\alpha, \beta}(x):=\left(\alpha+\frac{1}{2 \sigma^{2}}\right) x-\frac{\beta}{2} g^{\prime}(x) .
$$

The number of limit cycles in a Liénard system such as (10) mainly depends on the function $f_{\alpha, \beta}(x)$ : some tools to determine the exact number of limit cycles are available in literature (see [19], [5] and references therein) but their application may be cumbersome in a general setting, since several features of the function $f_{\alpha, \beta}(x)$ should be studied, such as the position of its zeroes, its local minima and maxima, their height and so on. However, playing with the form of the interaction function $g$, we can always create a Gaussian Curie-Weiss model with dissipation with a customized number of phase transitions and of coexisting limit cycles. In particular, from the results in [19], we can state the following.

Proposition 1 Fix $\sigma^{2}, \alpha>0$ and suppose that there exists a $\beta^{*}$ such that the following conditions are satisfied:

(i) the function $f_{\alpha, \beta^{*}}$ has $K$ positive zeros $x_{0}$ : $=0<x_{1}<\cdots<x_{K}\left(<x_{K+1}\right.$ a bound $)$ at which it changes sign; 
(ii) for every $k=1, \ldots, K$ there is a $C^{1}$ mapping $\phi_{k}:\left[x_{k-1}, x_{k}\right] \rightarrow\left[x_{k}, x_{k+1}\right]$ such that

$$
\phi_{k}(x) \phi_{k}^{\prime}(x) \geq x \text { and }\left|f_{\alpha, \beta^{*}}\left(\phi_{k}(x)\right)\right| \geq\left|f_{\alpha, \beta^{*}}(x)\right| ;
$$

(iii) the function $f_{\alpha, \beta^{*}}$ on each interval $\left[x_{k-1}, \phi_{k-1}\left(x_{k-1}\right)\right]$ for $2 \leq k \leq K+1$ has an extremum at a unique point $y_{k}$ and its derivative is weakly monotone.

Then the generalized Curie-Weiss model with dissipation has at least one regime in which it has exactly $N$ invariant sets of the form $\Gamma\left(\gamma_{i}\right)$ for some closed curves $\left\{\gamma_{i}\right\}_{i=1, \ldots, K}$. These are concentric cycles such that the outer is attractive, then the others alternate as repulsive and attractive, respectively.

The proof of this result is a simple application of the results in [19]. It is easy to see that the function $f_{\alpha, \beta}$ depends on the choice of the interaction function $g$. Since Assumptions 1 and 2 are not very restrictive, $g$ can be manipulated to obtain a system that admits a regime with the desired number of limit cycles.

\subsection{An Explicit Example}

With an explicit example, we show how we can manipulate the interaction function $g$ in order to observe the coexistence of two stable limit cycles. Let us define the function

$$
g(x)=\tanh \left(a x^{2}+b x^{4}+c x^{6}\right),
$$

with $a, b, c$ suitable constants such that $g$ stays strictly increasing on $[0, \infty)$. Fix $\sigma^{2}>0$, then the pair $(g, \rho)$, with $\rho \sim \mathcal{N}\left(0, \sigma^{2}\right)$ clearly satisfies Assumptions 1 and 2 and it defines a generalized Curie-Weiss model. We consider two triplets of constants $(1 / 2,-1,2)$ and $(1,1,0)$ in order to observe some particular regimes that do not exist for the classical CurieWeiss model with dissipation. Let us underline that, in the following, the qualitative study of the behavior of the process is made by means of the study of a system of ODE. We postpone to Sect. 3 the proof that this is indeed sufficient.

\subsubsection{Case A: Triplet $\left(\frac{1}{2},-1,2\right)$}

We see from Fig. 1 the changes in the concavity of $g^{\prime}(x)$. This causes an interesting behavior also in the dynamics without dissipation. Indeed, if we consider $\alpha=0$ and $\lambda_{0}=\left\langle v_{0}, x\right\rangle$, the dynamics in (7) is simply the mean field limit of the Langevin dynamics described in (2). This is a nonlinear process such that it suffices to study the following system

$$
\left\{\begin{array}{l}
\dot{m}_{t}=\frac{\beta}{2} g^{\prime}\left(m_{t}\right)-\frac{m_{t}}{2 \sigma^{2}}, \\
\dot{v}_{t}=1-\frac{v_{t}}{\sigma^{2}},
\end{array}\right.
$$

to analyze its stationary measures and its long-time behavior. We analyze in details only the equilibria of the first row in (13), since the two variables are independent. The ODE is characterized by three critical values of $\beta$ and the four following regimes:

- for $\beta<\beta_{1}$ the origin is a global attractor;

- for $\beta \in\left(\beta_{1}, \beta_{2}\right)$ the origin is locally stable, but there are four other equilibrium points $-x_{2}<-x_{1}<0<x_{1}<x_{2}$, such that $\pm x_{2}$ are stable and $\pm x_{1}$ are unstable;

- for $\beta \in\left(\beta_{2}, \beta_{3}\right)$ the origin becomes unstable and two additional stable equilibrium points appear, $\cdots-x_{1}<-x_{3}<0<x_{3}<x_{1} \ldots$; 

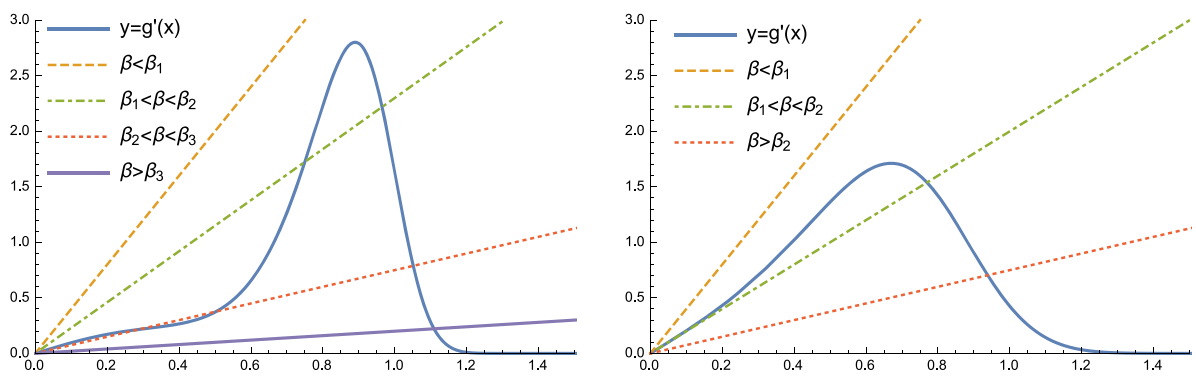

Fig. 1 The plot of the function $g^{\prime}$ and of lines $y=\frac{1}{\beta \sigma^{2}} x$ for different values of $\beta$. The number of intersections gives the number of equilibrium points in the positive axes. Left: the case A. Right: the case B

- for $\beta=\beta_{3}$ the pairs of equilibrium points $\left\{x_{3}, x_{1}\right\}$ and $\left\{-x_{3},-x_{1}\right\}$ collapse and disappear, such that for $\beta>\beta_{3}$ there are three equilibrium points $-x_{2}<0<x_{2}$, the outer two are stable and the origin is unstable.

The exact critical values may be obtained numerically, and the behavior of the dynamical system is clear from (13).

In this case, the dissipated dynamics (identified from the study of system (15)) actually shows four different regimes as well, but the critical values $\hat{\beta}_{1}(\alpha), \hat{\beta}_{2}(\alpha), \hat{\beta}_{3}(\alpha)$ are not straightforwardly obtained with the same procedure of the elements of $C V$. To be precise, if $\beta_{1}$ corresponds to the smallest value of $\beta$ in which the line $y=\frac{x}{\sigma^{2} \beta}$ is tangent to the graph of $y=g^{\prime}(x)$, the value $\hat{\beta}_{1}(\alpha)$ is strictly greater than the smallest value of $\beta$ such that the line $y=\frac{2 \alpha+\frac{1}{\sigma^{2}}}{\beta} x$ is tangent to the graph of $y=g^{\prime}(x)$. This means that there exists a $\beta^{*}$ such that the line $y=\frac{2 \alpha+\frac{1}{\sigma^{2}}}{\beta^{*}} x$ intersects the graph of $y=g^{\prime}(x)$ but any limit cycle occurs. Nevertheless, the system displays a regime of coexistence of stable limit cycles. Let us better explain the four regimes that we observe in system (15) (actually the computations and the plots refer to system (10), since the link with the function $f_{\alpha, \beta}$ is more clear in this case).

- For $\beta<\hat{\beta}_{1}(\alpha)$ the origin is a global attractor. Notice that, numerically we can see that $\hat{\beta}_{1}(\alpha)$ is greater that the $\beta^{*}$ obtained in Theorem 2 ; indeed it is not necessary that the function $f_{\alpha, \beta}(x)$ is strictly greater than zero for all $x>0$. It is reasonable to believe, see Fig. 2a, that for those $\beta$ such that the negative part of the function is "small enough" do not necessary give rise to a periodic orbit.

- For $\beta \in\left(\hat{\beta}_{1}(\alpha), \hat{\beta}_{2}(\alpha)\right)$ the origin is locally stable and, through a global bifurcation, two periodic orbits have arised, the larger one is stable and the smaller one is unstable. Indeed, we can prove numerically that there exists a value $\beta^{*} \in\left(\hat{\beta}_{1}(\alpha), \hat{\beta}_{2}(\alpha)\right)$ such that the function $f_{\alpha, \beta^{*}}$ satisfies the conditions of Theorem $\mathrm{A}$ and $\mathrm{B}$ in [19] for the existence of exactly two limit cycles. See Fig. $2 b$ in which we can observe the stable outer cycle and the attractivity of the origin.

- Notice that $\beta_{H}=\hat{\beta}_{2}(\alpha)$ from Theorem 2. Therefore, for $\beta \in\left(\hat{\beta}_{2}(\alpha), \hat{\beta}_{3}(\alpha)\right)$ we observe two attractive limit cycles, a smaller one spreading from the origin (that is now unstable) while the bigger one remains from the previous regime: see Fig. 2c. The basin of attraction of the two stable orbits are separated by a third unstable periodic orbit. This is the regime in which we see the coexistence of two stable periodic orbits, one inside the other. The existence of this regime is again a consequence of Theorem A and B in [19], because we 

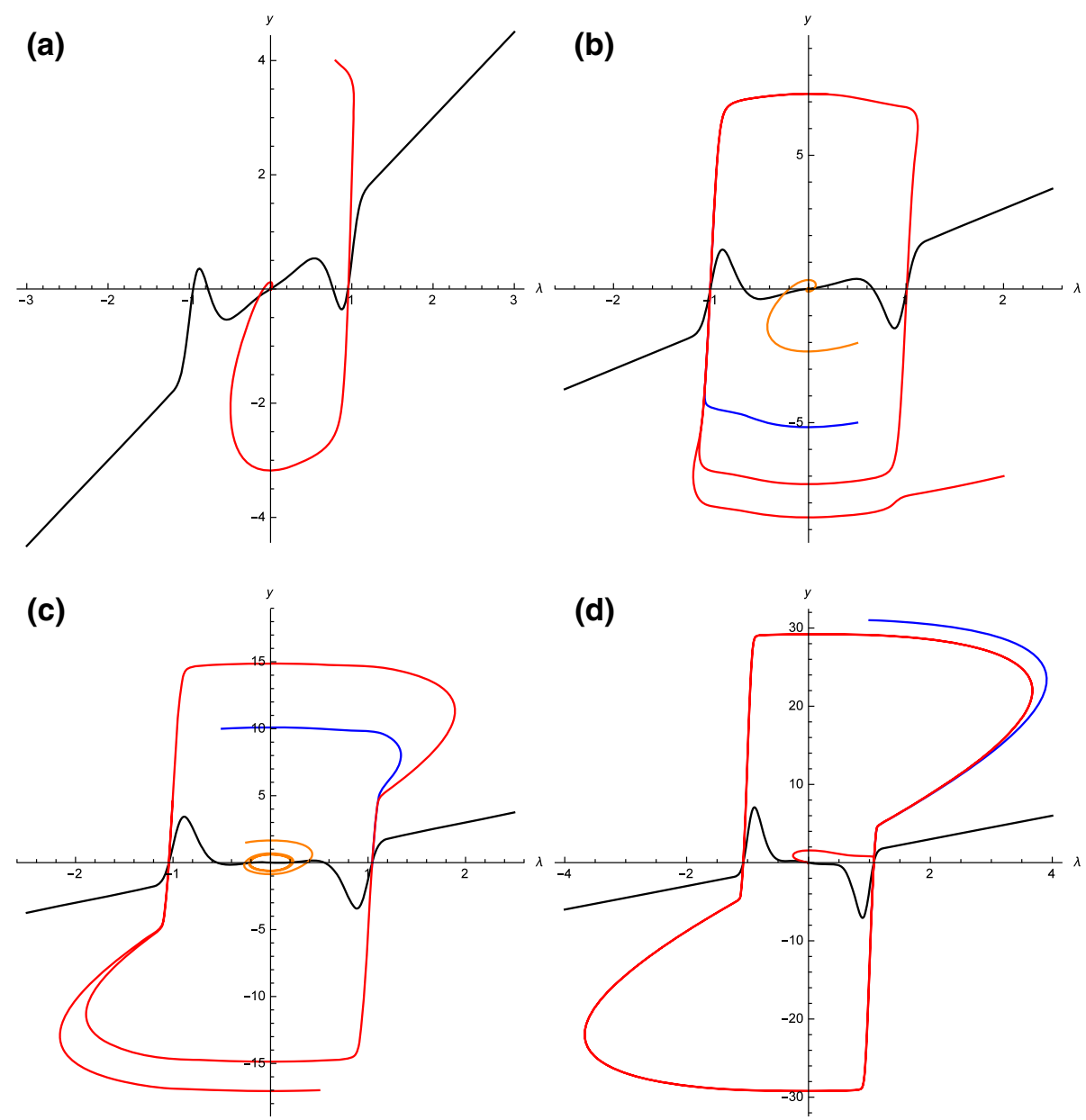

Fig. 2 Different regimes for case A. In all the pictures, the black line represents the graph of $y=f_{\alpha, \beta}(\lambda)$ and we fixed $\alpha=\sigma^{2}=1$. In a, the regime $\beta<\hat{\beta}_{1}(\alpha)(\beta=1.2)$ : the red line represents the solution starting from $\lambda(0)=1, y(0)=4$, which is definitely attracted by the globally stable origin. In $\mathbf{b}$, the regime $\beta \in\left(\hat{\beta}_{1}, \hat{\beta}_{2}\right)(\beta=2)$ : the red-colored solution, starting from $\lambda(0)=2, y(0)=-7$, and the blue-colored solution, starting from $\lambda(0)=0.5, y(0)=-5$, are attracted by a stable limit cycle. Here, the origin is locally stable (the orange-colored solution with initial condition $\lambda(0)=0.5, y(0)=-2$ is attracted by it) and its basin of attraction is surrounded by an unstable limit cycle. In $\mathbf{c}$, the regime $\beta \in\left(\hat{\beta}_{2}, \hat{\beta}_{3}\right)(\beta=3.4)$ : the red and blue lines, here representing solution starting from $\lambda(0)=0.5, y(0)=-17$ and $\lambda(0)=-0.5, y(0)=10$ respectively, are again attracted by the outer cycle but now the origin is unstable and another stable cycle is born via the Hopf bifurcation. The orange-colored solution, with initial condition $\lambda(0)=-0.25, y(0)=1.5$, is attracted by the smallest cycle. The basins of attraction of the stable orbits is separated by an unstable cycle. In $\mathbf{d}$, the regime $\beta>\hat{\beta}_{3}(\beta=6)$ : only the external orbit is survived and it has become globally attractive, as shown by the red and blue lines, with initial conditions $\lambda(0)=0, y(0)=-0.005$ and $\lambda(0)=1.5, y(0)=31$ respectively

can numerically find a $\beta^{* *}$ that satisfies the hypothesis for the existence of exactly three limit cycles, two stable and one unstable.

- For $\beta>\hat{\beta}_{3}(\alpha)$ we see that only the largest periodic orbit has survived. Indeed, for in $\hat{\beta}_{3}(\alpha)$ the smallest stable orbit and the unstable one collapse and disappear. Of course, 

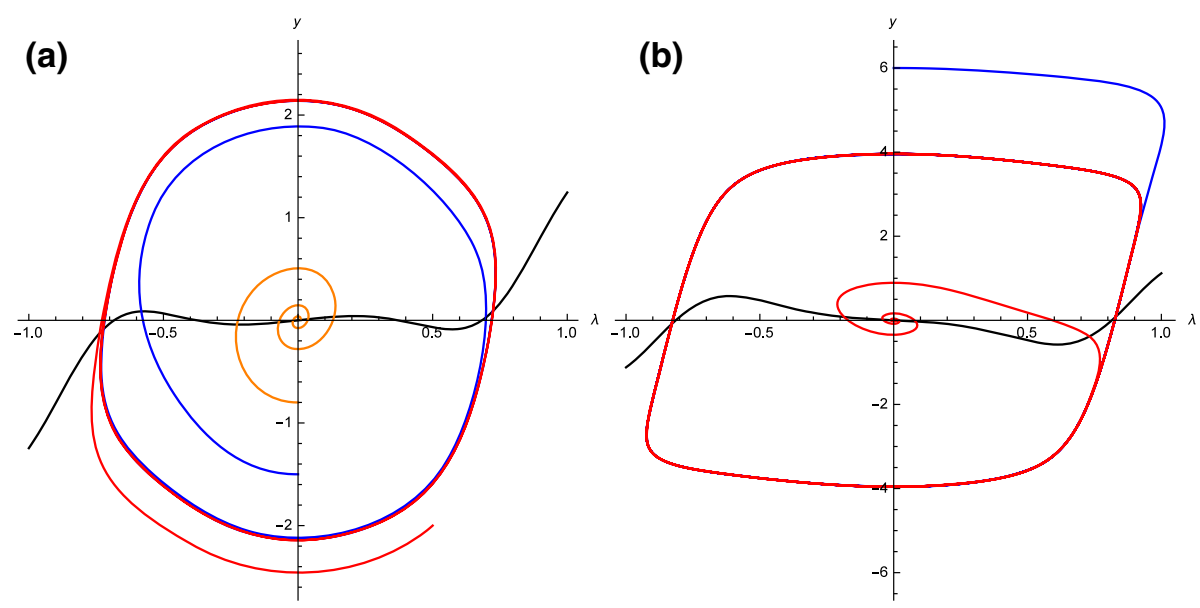

Fig. 3 Different regimes for case B close to the Hopf bifurcation. In both pictures, the black line represents the graph of $y=f_{\alpha, \beta}(\lambda)$ and we fixed $\alpha=\sigma^{2}=1$. In a, the regime $\beta \in\left(\hat{\beta}_{1}(\alpha), \beta_{H}\right)(\beta=1.2)$ : the situation is qualitatively the same of Fig. $2 \mathrm{~b}$. The red, blue and orange lines represent solution starting from $\lambda(0)=0.5$, $y(0)=-2, \lambda(0)=0, y(0)=-1.5$ and $\lambda(0)=0, y(0)=-0.8$ respectively. In $\mathbf{b}$, the regime $\beta>\beta_{H}$ $(\beta=1.8)$ : the system has undergone through a Hopf bifurcation but the stable limit cycle spreading from the origin is not present here, due to the collapse of the unstable cycle in the origin, leaving the outer orbit to become globally attractive. The red-colored and blue-colored solutions have initial conditions $\lambda(0)=0$, $y(0)=-0.005$ and $\lambda(0)=0, y(0)=6$ respectively

we see that this $\hat{\beta}_{3}(\alpha)=\beta_{U C}$ defined in Theorem 2; but from numerical evidence we suppose that for this value of $\beta$ the function $f_{\alpha, \beta}$ has more than one single zero in the positive half-line, but that the other two zeros are not distant enough to admit the existence of the two inner orbits. Of course when $\beta$ is such that there exists a unique positive zero for $f_{\alpha, \beta}$, we analitically prove the existence and uniqueness of the limit cycle (see Theorem 2) while for lower values we can only show it numerically, see Fig. $2 \mathrm{~d}$.

\subsubsection{Case B: Triplet $(1,1,0)$}

We see in Fig. 1 that the shape of $g^{\prime}$ basically allows three different regimes for the case without dissipation. Indeed we have $\beta_{1}<\beta_{2}=\frac{2}{\sigma^{2}}$ and the three regimes are the following:

- for $\beta<\beta_{1}$ the origin is a global attractor;

- for $\beta \in\left(\beta_{1}, \beta_{2}\right)$ there are five equilibrium points $-x_{2}<-x_{1}<0<x_{1}<x_{2}$, where $\pm x_{1}$ are unstable, while the others are stable;

- at $\beta=\beta_{1}$ the two points $\pm x_{1}$ collapse in the origin that becomes unstable, such that for $\beta>\beta_{1}$ the origin is unstable and the points $\pm x_{2}$ are stable.

We treat this example in the dissipated case (15) (by means of the Liénard system (10)). We expect three regimes and, in particular, we will observe an atypical behavior at the Hopf bifurcation, where we will not have a small limit cycle bifurcating from the origin, but the already existing stable limit cycle that becomes a global attractor. In Fig. 3 we compare the regimes immediately below and above the Hopf bifurcation.

- For $\beta<\beta_{1}(\alpha)$, the origin is a global attractor. As is Case A the value $\beta_{1}(\alpha)$ is strictly greater than the value in which the line first touches the graph $y=g^{\prime}(x)$. 
- For $\beta \in\left(\beta_{1}(\alpha), \beta_{H}\right)$ the origin is stable and we have an unstable periodic orbit contained in a stable periodic one. When $\beta$ increase the inner orbit shrinks and the outer expands.

- For $\beta=\beta_{H}$ the Hopf bifurcation is such that the origin looses stability, but this happens simultaneously to the collapse of the unstable periodic orbit on it. Therefore, after the bifurcation, we do not see the usual periodic orbit expanding form the origin because the unique orbit is the stable one (from the previous regime) that becomes globally stable.

This case is interesting because the Hopf bifurcation does not originate a small periodic orbit. However, the phenomenon is still a local one, because it is a small unstable orbit that collapses on the origin changing its stability.

\section{Proof of Theorem 1}

As we mentioned before stating Theorem 1, under Assumptions 1 and 2, the law $\mu_{t}=$ $\operatorname{Law}\left(X_{t}, \lambda_{t}\right)$ of the solution of (7) has the product form

$$
\mu_{t}(d x, d \lambda)=v_{t}(d x) \times \delta_{\lambda_{t}}(d \lambda)
$$

Let $m_{t}=\left\langle\mu_{t}(d x, d l), x\right\rangle$ and $v_{t}=\operatorname{Var}\left[X_{t}\right]$, then the equations describing the time evolution of the quantities $\left\{\left(m_{t}, v_{t}, \lambda_{t}\right)\right\}_{t \geq 0}$ are the following:

$$
\left\{\begin{array}{l}
\dot{m}_{t}=\frac{\beta}{2} g^{\prime}\left(\lambda_{t}\right)-\frac{m_{t}}{2 \sigma_{2}}, \\
\dot{v}_{t}=1-\frac{v_{t}}{\sigma^{2}}, \\
\dot{\lambda_{t}}=-\alpha \lambda_{t}+\frac{\beta}{2} g^{\prime}\left(\lambda_{t}\right)-\frac{m_{t}}{2 \sigma^{2}} .
\end{array}\right.
$$

These equations are closed and they describe the whole behavior of the process when $v_{0}$ is a Gaussian distribution (and the process is consequently a Gaussian process). Moreover, the study of the system (14) and of its periodic orbits is sufficient to identify periodic solutions and long time behavior of (7), as long as Assumptions 1 and 2 hold. Under these assumptions, periodic solutions have precisely a Gaussian structure, while other solutions approach a Gaussian shape.

In the following, we will therefore focus on system (14). By the independence of the evolution of $v_{t}$, we study the two-dimensional system

$$
\left\{\begin{array}{l}
\dot{m}_{t}=\frac{\beta}{2} g^{\prime}\left(\lambda_{t}\right)-\frac{m_{t}}{2 \sigma_{2}}, \\
\dot{\lambda_{t}}=-\alpha \lambda_{t}+\frac{\beta}{2} g^{\prime}\left(\lambda_{t}\right)-\frac{m_{t}}{2 \sigma^{2}} .
\end{array}\right.
$$

instead of the three-dimensional (14). By the change of variable $y=\frac{1}{2 \sigma^{2}}(\lambda-m)$ we see that (15) is equivalent to the Liénard system (10) and we study its behavior.

Theorem 2 Fix $\sigma^{2}>0$ and $\alpha>0$ and consider the dynamical system (10) under Assumptions 1 and 2.

(i) There exists $\beta^{*}>0$ such that $\forall \beta \in\left(0, \beta^{*}\right)$ the origin is a global attractor for (10).

(ii) If $g^{\prime \prime}(0)>0$ and $g^{\prime}$ is not linear in 0 , the origin looses stability via a Hopf bifurcation at the critical value $\beta_{H}=\frac{2 \alpha+\frac{1}{\sigma^{2}}}{g^{\prime \prime}(0)} \geq \beta^{*}$.

(iii) If $g^{\prime \prime}(0)>0$ and there exists $C>0$ such that for all $x \in(C, \infty)$ the function $g^{\prime}(x)$ is concave, then there exists a $\beta_{U C} \geq \beta_{H}$ such that for all $\beta>\beta_{U C}$ there exists a unique limit cycles for (10). 
Proof (i) Let us consider the function

$$
W(y, \lambda)=\frac{\alpha}{4 \sigma^{2}} \lambda^{2}+\frac{y^{2}}{2},
$$

it is clear that

$$
\frac{d}{d t} W(y(t), \lambda(t))=-\frac{\alpha}{2 \sigma^{2}} \lambda\left(\left(\alpha+\frac{1}{2 \sigma^{2}}\right) \lambda-\frac{\beta}{2} g^{\prime}(\lambda)\right) .
$$

We are left to consider the intersection of the graph of the function $y=g^{\prime}(\lambda)$ with the line $y=\frac{2 \alpha+\frac{1}{\sigma^{2}}}{\beta} \lambda$. We see that there exists a $\beta^{*}$ sufficiently small, such that $\forall \beta<\beta^{*}$ the only intersection is the origin, meaning that (16) is strictly negative except than at $(0,0)$, in which it is zero. Therefore $W$ is a global Lyapunov function, proving global attractivity of the origin.

(ii) We consider the system (10) linearized around the point $(0,0)$, that gives the linear system:

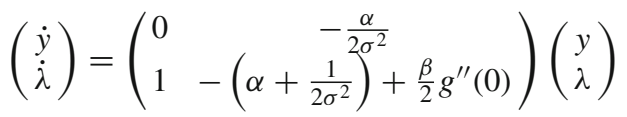

with eigenvalues

$$
x_{ \pm}=\frac{1}{2}\left(\frac{\beta}{2} g^{\prime \prime}(0)-\alpha-\frac{1}{2 \sigma^{2}} \pm \sqrt{\left(\frac{\beta}{2} g^{\prime \prime}(0)-\alpha-\frac{1}{2 \sigma^{2}}\right)^{2}-\frac{2 \alpha}{\sigma^{2}}}\right) .
$$

When $\beta=\beta_{H}$, the eigenvalues $x_{ \pm}$constitute a pair of conjugate non-zero purely imaginary numbers, crossing the imaginary line with positive velocity. The assumption on $g^{\prime}$ being not linear around 0 assures that we are in presence of a local bifurcation, and we can conclude that when $\beta=\beta_{H}$ we have a Hopf bifurcation. Clearly $\beta_{H} \geq \beta^{*}$ because the origin is globally stable for all $\beta<\beta^{*}$.

(iii) Recall that under Assumption $1, g^{\prime}$ is an odd function positive on $(0, \infty)$. Moreover, by Assumption 2, in the Gaussian case $g^{\prime}$ has to be definitively sub-linear (it is clear by (6)). If, in addition, $g^{\prime \prime}(0)>0$ and there exists $C>0$ such that for all $x \in(C, \infty)$ the function $g^{\prime}$ is concave, then there exists a $\beta_{U C} \geq \beta_{H}$ sufficiently large such that $\forall \beta>\beta_{U C}$, the function $f_{\alpha, \beta}$ has exactly three zeros $-x^{*}<0<x^{*}$ and satisfies the following: $f_{\alpha, \beta}$ is negative on $\left(0, x^{*}\right)$ and positive and monotonically increasing on $\left(x^{*}, \infty\right)$. In this way, for all $\beta>\beta_{U C}$, the system (10) satisfies the conditions for the existence and uniqueness of a limit cycle presented in Theorem 1.1 of [3]. The proof follows the usual approach for Liénard systems, used also in [9].

First it is shown that the $y$ axis and the function $y=f_{\alpha, \beta}(\lambda)$ divides the $(\lambda, y)$-plane in four regions:

$$
\begin{aligned}
& I \doteq\left\{(\lambda, y): \lambda>0 ; y>f_{\alpha, \beta}(\lambda)\right\} ; \\
& I I \doteq\left\{(\lambda, y): \lambda>0 ; y<f_{\alpha, \beta}(\lambda)\right\} \text {; } \\
& I I I \doteq\left\{(\lambda, y): \lambda<0 ; y<f_{\alpha, \beta}(\lambda)\right\} \text {; } \\
& I V \doteq\left\{(\lambda, y): \lambda<0 ; y>f_{\alpha, \beta}(\lambda)\right\} \text {. }
\end{aligned}
$$

In each of these four regions the vector field pushes the trajectories to cross either the $y$ axis or the graph $y=f_{\alpha, \beta}(\lambda)$. Therefore each trajectory is forced to revolve clockwise around the origin. 
Then, for $y_{0}>0$, we consider a trajectory starting from the point $\left(0, y_{0}\right)$ and we call $y_{1}>0$ its first intersection with the $y$-axis in the negative half-plane. We define the function

$$
\Delta W\left(y_{0}\right)=W\left(0, y_{1}\right)-W\left(0, y_{0}\right) .
$$

Of course, when $\Delta W(\bar{y})=0$, the trajectory starting from $(0, \bar{y})$ is a periodic orbit. Let $y_{0}^{*}>0$ be such that the trajectory starting at time zero from $\left(0, y_{0}^{*}\right)$ passes through $\left(x^{*}, 0\right)$, the positive zero of the function $f_{\alpha, \beta}(\lambda)$. Then, let us define $t^{*}$ the time in which this trajectory crosses the $y$-axis, this time it must be $y\left(t^{*}\right)<0$, by the above observation. Notice that, with this notation and by (16), we have that

$$
\Delta W\left(y^{*}\right)=\int_{0}^{t^{*}} \frac{d}{d t} W(y(t), \lambda(t)) d t=-\int_{0}^{t^{*}} \frac{\alpha}{2 \sigma^{2}} \lambda(t) f_{\alpha, \beta}(\lambda(t)) d t .
$$

This trajectory is such that, for all $t \in\left[0, t^{*}\right]$, we have that $\lambda(t) \in\left[0, x^{*}\right]$, since in $\left(x^{*}, 0\right)$ it moves from the region $I$ of the plane to the region $I I$, in which the $\lambda$-coordinate starts to decrease. Moreover, it is clear that, for all $\lambda \in\left(0, x^{*}\right)$ the function $\lambda f_{\alpha, \beta}(\lambda)$ is strictly negative, due to the sign of $f_{\alpha, \beta}$, that is the reason why the integral in (17) is strictly positive. Clearly, the same holds for all the trajectories closer than this one to the origin, i.e. $\Delta W(y)>0$ for all $y<y^{*}$. Then, since the function $f_{\alpha, \beta}(\lambda)$ remains positive for all $\lambda>x^{*}$, we see that $\Delta W(y)$ decreases monotonically to $-\infty$, when $y \rightarrow \infty$, meaning that there exists a unique $\bar{y}$ for which it is zero.

Now, we aim to extend the results on the dynamical system (15), to the stochastic process itself, by using the result of Theorem 2 to prove Theorem 1 .

Proof (Theorem 1) Let us restrict to the class of measures of the form $\mu(d x, d l)=v(d x) \times$ $\delta_{\lambda}(d l)$, for $v \in \mathcal{M}(\mathbb{R})$ and $\lambda \in \mathbb{R}$. Suppose that there exist a stationary measure for (7) in this class, call it $\mu^{*}(d x, d l)=v^{*}(d x) \times \delta_{\lambda^{*}}(d l)$, then it must satisfy

$$
\left\{\begin{array}{l}
0=\frac{1}{2} \frac{d^{2}}{d x^{2}} \nu^{*}(x)-\frac{d}{d x}\left[\left(\frac{\beta}{2} g^{\prime}\left(\lambda^{*}\right)-\frac{x}{2 \sigma^{2}}\right) v^{*}(x)\right] \\
0=-\alpha \lambda^{*}+\frac{\beta}{2} g^{\prime}\left(\lambda^{*}\right)-\frac{m^{*}}{2 \sigma^{2}}
\end{array}\right.
$$

with $m^{*}=\int_{\mathbb{R}} x v^{*}(x) d x$. Let us solve the first row in (18), we see that there exists $K \in \mathbb{R}$ such that

$$
\frac{d}{d x} v^{*}(x)=K+\left(\frac{\beta}{2} g^{\prime}\left(\lambda^{*}\right)-\frac{x}{2 \sigma^{2}}\right) v^{*}(x) .
$$

Thus $v^{*}(x)$ solves a linear ODE, i.e. there exists $C \in \mathbb{R}$ such that

$$
\nu^{*}(x)=\exp \left(\beta g^{\prime}\left(\lambda^{*}\right) x-\frac{x^{2}}{2 \sigma^{2}}\right)\left(C+K \int_{-\infty}^{x} \exp \left(-\beta g^{\prime}\left(\lambda^{*}\right) y+\frac{y^{2}}{2 \sigma^{2}}\right) d y\right) .
$$

Let us define the values of the constants:

- $K=0$, because if $K \neq 0 v^{*}(x)$ is not integrable;

- $C=\int_{\mathbb{R}} \exp \left(\beta g^{\prime}\left(\lambda^{*}\right) x-\frac{x^{2}}{2 \sigma^{2}}\right) d x$, such that $\nu^{*}$ has mass one.

The admissible functions $v^{*}$ are such that

$$
m^{*}=\int_{\mathbb{R}} x v^{*}(x) d x=\beta \sigma^{2} g^{\prime}\left(\lambda^{*}\right),
$$

but they must simultaneously satisfy the second row in (18), then the only solution is such that $\left(m^{*}, \lambda^{*}\right)=(0,0)$ and this gives the thesis. 
Now, let us prove the long-time behavior of $\mu_{t}$ for any square-integrable initial condition of the type $\mu_{0}=v_{0} \times \delta_{\lambda_{0}}$. As we said, this implies that $\mu_{t}=v_{t} \times \delta_{\lambda_{t}}$ for all $t>0$. To this aim we introduce the following time-inhomogeneous SDE:

$$
\left\{\begin{array}{l}
d Y_{t}=\frac{\beta}{2} g^{\prime}\left(\lambda_{t}\right) d t-\frac{Y_{t}}{2 \sigma^{2}} d t+d B_{t} \\
Y_{0} \in L^{2}(\Omega)
\end{array}\right.
$$

where $\lambda_{t}$ solves (15) with initial condition $\left(m_{0}, \lambda_{0}\right)=\left(\left\langle x, v_{0}\right\rangle, \lambda_{0}\right)$. The solution of (20) will be used as an auxiliary process to prove long-behavior of the solution of (7). Indeed it is clear that, for all $t \geq 0, X_{t}$ in (7) has the same law of $Y_{t}$ in (20), provided that $v_{0}=\operatorname{Law}\left(Y_{0}\right)$. In the following Lemma we prove that, if $\left(\left\langle x, v_{0}\right\rangle, \lambda_{0}\right)$ belongs to the domain of attraction of a certain closed subset $\gamma \in \mathbb{R}^{2}$ (being that an equilibrium point or a closed orbit), then the law of $Y_{t}$ approaches a Gaussian law with mean $m^{*}$ and variance $\sigma^{2}$, where $\left(m^{*}, \lambda^{*}\right) \in \gamma$ for a certain $\lambda^{*}$.

Lemma 1 Let $\left\{Y_{t}\right\}$ be the solution of $(20)$, let $P_{t}\left(Y_{0}, \cdot\right)$ be its law and let $\left(m_{0}, \lambda_{0}\right)$ belong to the domain of attraction of $\gamma$ according to the dynamics (15). Then,

$$
\lim _{t \rightarrow+\infty} \inf _{(a, b) \in \gamma}\left\|P_{t}\left(Y_{0}, \cdot\right)-v_{a}(\cdot)\right\|_{T V}=0
$$

where $v_{a}(d x)=\frac{1}{\sqrt{2 \pi \sigma^{2}}} e^{\frac{(x-a)^{2}}{2 \sigma^{2}}} d x$.

Proof (Proof of Lemma 1) First of all, notice that

$$
\lim _{t \rightarrow+\infty} \inf _{(a, b) \in \gamma}\left|\int_{0}^{t} e^{\frac{s-t}{2 \sigma^{2}}} \frac{\beta}{2} g^{\prime}\left(\lambda_{s}\right) d s-a\right|=0 .
$$

In fact, fix $t>0$, and $(a, b) \in \gamma$ then

$$
\begin{aligned}
\int_{0}^{t} e^{\frac{s-t}{2 \sigma^{2}}} \frac{\beta}{2} g^{\prime}\left(\lambda_{s}\right) d s-a= & \int_{0}^{t} e^{\frac{s-t}{2 \sigma^{2}}} \frac{\beta}{2} g^{\prime}\left(\lambda_{s}\right) d s-e^{-\frac{t}{2 \sigma^{2}}} \int_{0}^{t} e^{\frac{s}{2 \sigma^{2}}} \frac{m_{s}}{2 \sigma^{2}} d s \\
& +e^{-\frac{t}{2 \sigma^{2}}} \int_{0}^{t} e^{\frac{s}{2 \sigma^{2}}} \frac{m_{s}}{2 \sigma^{2}} d s-a \\
= & \int_{0}^{t} e^{\frac{s-t}{2 \sigma^{2}}} \dot{m}_{s} d s+e^{-\frac{t}{2 \sigma^{2}}} \int_{0}^{t} e^{\frac{s}{2 \sigma^{2}}} \frac{m_{s}}{2 \sigma^{2}} d s-a \\
= & m_{t}-a-e^{-\frac{t}{2 \sigma^{2}}} m_{0} .
\end{aligned}
$$

Since $\left(m_{0}, \lambda_{0}\right)$ belongs to the domain of attraction of $\gamma$, this implies (21). Then, if $\mu_{0}(\cdot)=$ $\operatorname{Law}\left(Y_{0}\right)$,

$$
\begin{gathered}
\inf _{(a, b) \in \gamma}\left\|P_{t}\left(Y_{0}, \cdot\right)-v_{a}(\cdot)\right\|_{T V}=\inf _{(a, b) \in \gamma} \int_{\mathbb{R}}\left\|P_{t}(y, \cdot)-v_{a}(\cdot)\right\|_{T V} d v_{0}(y) \\
=\inf _{(a, b) \in \gamma} \frac{1}{2} \int_{\mathbb{R}} \int_{\mathbb{R}}\left|\frac{\left.\exp \mid \frac{\left(x-y e^{-\frac{t}{2 \sigma^{2}}}-\int_{0}^{t} e^{\left.\frac{s-t}{2 \sigma^{2}} \frac{\beta}{2} g^{\prime}\left(\lambda_{s}\right) d s\right)^{2}}\right.}{2 \sigma^{2}\left(1-e^{-\frac{t}{2 \sigma^{2}}}\right)}\right)}{\sqrt{2 \pi \sigma^{2}\left(1-e^{-\frac{t}{2 \sigma^{2}}}\right)}}-\frac{e^{\frac{(x-a)^{2}}{2 \sigma^{2}}}}{\sqrt{2 \pi \sigma^{2}}}\right| d x d \nu_{0}(y)
\end{gathered}
$$

which converges to 0 as $t \rightarrow+\infty$ thanks to (21). 
As we said, if $\nu_{0}=\operatorname{Law}\left(Y_{0}\right), X_{t}$ in (7) has the same law of $Y_{t}$ in (20) for all $t \geq 0$. Then the claims follow directly from Theorem 2 and Lemma 1.

\section{Proof of the McKean-Vlasov Limit}

In this section we collect the standard proofs of well-posedness of the nonlinear Markov process (5) and we identify its law as the weak limit of the sequence of empirical measures of (3). Indeed, in (3) we describe the time-evolution of a stochastic process $\left(X^{N}(t), \lambda^{N}(t)\right)_{t>0}$ with values in $\mathbb{R}^{2 N}$ where, at every time $t \geq 0, X^{N}(t)=\left(X_{1}^{N}(t), \ldots, X_{N}^{N}(t)\right)$ is the vector of the spins of the $N$ particles and $\lambda^{N}=\left(\lambda_{1}^{N}(t), \ldots, \lambda_{N}^{N}(t)\right)$ is the vector of the "perceived magnetizations". The Markov process $\left(X^{N}, \lambda^{N}\right)$ has infinitesimal generator

$$
\begin{aligned}
\mathcal{L}^{N} f(x, \lambda) & =\sum_{i=1}^{N}\left[\frac{1}{2}\left(\beta g^{\prime}\left(\lambda_{i}\right)+\frac{\rho^{\prime}\left(x_{i}\right)}{\rho\left(x_{i}\right)}\right) \frac{\partial}{\partial x_{i}} f(x, \lambda)+\frac{1}{2} \frac{\partial^{2}}{\partial x_{i}^{2}} f(x, \lambda)\right. \\
& +\frac{1}{2 N} \sum_{j=1}^{N} \frac{\partial^{2}}{\partial x_{i} \partial \lambda_{j}} f(x, \lambda)+\left(\frac{1}{2 N} \sum_{j=1}^{N}\left(\beta g^{\prime}\left(\lambda_{j}\right)+\frac{\rho^{\prime}\left(x_{j}\right)}{\rho\left(x_{j}\right)}\right)-\alpha \lambda_{i}\right) \frac{\partial}{\partial \lambda_{i}} f(x, \lambda) \\
& \left.+\frac{D}{2} \frac{\partial^{2}}{\partial \lambda_{i}^{2}} f(x, \lambda)+\frac{1}{2 N} \sum_{j=1}^{N} \frac{\partial^{2}}{\partial x_{j} \partial \lambda_{i}} f(x, \lambda)+\frac{1}{2 N} \sum_{j=1}^{N} \frac{\partial^{2}}{\partial \lambda_{j} \partial \lambda_{i}} f(x, \lambda)\right]
\end{aligned}
$$

We associate to (3) the nonlinear Markov process described in (5), which is commonly called the McKean-Vlasov limit of the particle system (3), see [21] and reference therein. In the following theorems we prove well-posedness of the nonlinear process and the so-called property of propagation of chaos of the particle system (3).

Theorem 3 The nonlinear process (5) is well-defined, i.e. there exists a unique strong solution for all square-integrable initial condition $\left(X_{0}, \lambda_{0}\right) \in \mathbb{R}^{2}$.

Theorem 4 Let $\left(X^{N}(t), \lambda^{N}(t)\right)_{t \geq 0}$ be the Markov process with generator (22) starting from i.i.d. initial conditions Law $\left(\left(X_{i}^{N}, \lambda_{i}^{N}\right)\right)=\mu_{0}$ on $\mathbb{R}^{2}$, where $\int_{\mathbb{R}^{2}}\left(x^{2}+\lambda^{2}\right) \mu_{0}(d x, d \lambda)<\infty$, and denote with $P^{N}$ its law on $\mathbf{C}\left([0, T], \mathbb{R}^{2 N}\right)$. Let $(X(t), \lambda(t))_{t \geq 0}$ be the solution to (5) with initial condition $\mu_{0}$, and denote with $\mu$ its law on $\mathbf{C}\left([0, T], \mathbb{R}^{2}\right)$. Then, the sequence $\left(P^{N}\right)_{N \in \mathbb{N}}$ is $\mu$-chaotic.

The proofs of Theorem 3 and 4 follow a standard approach via pathwise estimates. To this aim, let us introduce a suitable distance on the space of trajectories. We fix a time $T>0$ and we define the $W_{2}$ Wasserstein distance on the set $\mathcal{M}^{2}\left(\mathbf{C}\left([0, T], \mathbb{R}^{2}\right)\right)$ of square-integrable measures: for all $\mu, v \in \mathcal{M}^{2}\left(\mathbf{C}\left([0, T], \mathbb{R}^{2}\right)\right)$

$$
\begin{aligned}
& D_{2, T}(\mu, v)^{2}=\inf \left\{\int \sup _{t \in[0, T]}\|x(s)-y(s)\|^{2} m(d x, d y),\right. \\
& \text { with } \left.m \in \mathcal{M}^{2}\left(\mathbf{C}\left([0, T], \mathbb{R}^{2}\right) \times \mathbf{C}\left([0, T], \mathbb{R}^{2}\right)\right), \pi_{1} \circ m=\mu, \pi_{2} \circ m=v\right\} .
\end{aligned}
$$

Proof (Proof of Theorem 3) Given any square-integrable law $\mu_{0}$ on $\mathbb{R}^{2}$, we define a map $\Gamma$ that associates to a measure $Q \in \mathcal{M}^{2}\left(\mathbf{C}\left([0, T], \mathbb{R}^{2}\right)\right)$ the law of the solution $\left\{\left(X_{t}, \lambda_{t}\right)\right\}_{t \in[0, T]}$ of the SDE 


$$
\left\{\begin{array}{l}
d X_{t}=\frac{\beta}{2} g^{\prime}\left(\lambda_{t}\right) d t+\frac{\rho^{\prime}\left(X_{t}\right)}{2 \rho\left(X_{t}\right)} d t+d B_{t}^{1} \\
d \lambda_{t}=-\alpha \lambda_{t} d t+\left\langle Q_{t}(d x, d l), \frac{\beta}{2} g^{\prime}(l)+\frac{\rho^{\prime}(x)}{2 \rho(x)}\right\rangle d t+D d B_{t}^{2}
\end{array}\right.
$$

that, for $\mu_{0}$ initial condition, admits a unique strong solution for classical results, see [17]; of course a solution to (5) is a fixed point of $\Gamma$. We use a coupling argument to prove existence (via a Picard iteration) and uniqueness of the fixed point of $\Gamma$. Let us start with the proof of uniqueness, if $Q^{1}$ and $Q^{2}$ are two fixed point of $\Gamma$, i.e. two measures in $\mathcal{M}^{2}\left(\mathbf{C}\left([0, T], \mathbb{R}^{2}\right)\right)$ such that $Q^{1}=\Gamma\left(Q^{1}\right)$ and $Q^{2}=\Gamma\left(Q^{2}\right)$. We couple them as follows. Let $\left(\Omega, \mathcal{F},\left\{\mathcal{F}_{t}\right\}_{t \in[0, T]}, \mathbf{P}\right)$ be a filtered probability space and $\left\{B_{t}\right\}_{t \in[0, T]}$ a two-dimensional Brownian motion. Then we write

$$
\left\{\begin{array}{l}
d X_{t}^{1}=\frac{\beta}{2} g^{\prime}\left(\lambda_{t}^{1}\right) d t+\frac{\rho^{\prime}\left(X_{t}^{1}\right)}{2 \rho\left(X_{t}^{1}\right)} d t+d B_{t}^{1} \\
d \lambda_{t}^{1}=-\alpha \lambda_{t}^{1} d t+\left\langle Q_{t}^{1}(d x, d l), \frac{\beta}{2} g^{\prime}(l)+\frac{\rho^{\prime}(x)}{2 \rho(x)}\right\rangle d t+D d B_{t}^{2},
\end{array}\right.
$$

and

$$
\left\{\begin{array}{l}
d X_{t}^{2}=\frac{\beta}{2} g^{\prime}\left(\lambda_{t}^{2}\right) d t+\frac{\rho^{\prime}\left(X_{t}^{2}\right)}{2 \rho\left(X_{t}^{2}\right)} d t+d B_{t}^{1} \\
d \lambda_{t}^{2}=-\alpha \lambda_{t}^{2} d t+\left\langle Q_{t}^{2}(d x, d l), \frac{\beta}{2} g^{\prime}(l)+\frac{\rho^{\prime}(x)}{2 \rho(x)}\right\rangle d t+D d B_{t}^{2},
\end{array}\right.
$$

where the initial conditions are $\left(X_{0}^{1}, \lambda_{0}^{1}\right)=\left(X_{0}^{2}, \lambda_{0}^{2}\right)$ a.s., $\mu_{0}$ distributed. We estimate the distance between $Q^{1}$ and $Q^{2}$ by means of the above coupling, i.e.

$$
D_{2, T}\left(Q^{1}, Q^{2}\right) \leq \sqrt{\mathbf{E}\left[\sup _{t \in[0, T]}\left(X_{t}^{1}-X_{t}^{2}\right)^{2}+\left(\lambda_{t}^{1}-\lambda_{t}^{2}\right)^{2}\right]} .
$$

The SDE for $\lambda^{1}$ and $\lambda^{2}$ is linear, then we write explicitly

$$
\lambda_{t}^{1}-\lambda_{t}^{2}=\int_{0}^{t} e^{\alpha(s-t)}\left\langle Q_{s}^{1}(d x, d l)-Q_{s}^{2}(d x, d l), \frac{\beta}{2} g^{\prime}(l)+\frac{\rho^{\prime}(x)}{2 \rho(x)}\right\rangle d s,
$$

but notice that $\left\langle Q_{t}^{1}(d x, d l)-Q_{t}^{2}(d x, d l), \frac{\beta}{2} g^{\prime}(l)+\frac{\rho^{\prime}(x)}{2 \rho(x)}\right\rangle=\frac{d}{d t} \mathbf{E}\left[X_{t}^{1}-X_{t}^{2}\right]$, that gives

$$
\lambda_{t}^{1}-\lambda_{t}^{2}=\mathbf{E}\left[X_{t}^{1}-X_{t}^{2}\right]-\alpha \int_{0}^{t} \mathbf{E}\left[X_{s}^{1}-X_{s}^{2}\right] e^{-\alpha(t-s)} d s .
$$

We use Ito's formula to obtain

$$
\left(X_{t}^{1}-X_{t}^{2}\right)^{2}=2 \int_{0}^{t}\left(X_{s}^{1}-X_{s}^{2}\right)\left(\frac{\beta}{2} g^{\prime}\left(\lambda_{s}^{1}\right)-\frac{\beta}{2} g^{\prime}\left(\lambda_{s}^{2}\right)+\frac{\rho^{\prime}\left(X_{s}^{1}\right)}{2 \rho\left(X_{s}^{1}\right)}-\frac{\rho^{\prime}\left(X_{s}^{2}\right)}{2 \rho\left(X_{s}^{2}\right)}\right) d s .
$$

Therefore, there exists $C_{T}$ such that

$\mathbf{E}\left[\sup _{t \in[0, T]}\left(X_{t}^{1}-X_{t}^{2}\right)^{2}+\left(\lambda_{t}^{1}-\lambda_{t}^{2}\right)^{2}\right] \leq C_{T} \int_{0}^{T} \mathbf{E}\left[\sup _{t \in[0, s]}\left(X_{t}^{1}-X_{t}^{2}\right)^{2}+\left(\lambda_{t}^{1}-\lambda_{t}^{2}\right)^{2}\right] d s$,

and by Gronwall Lemma this gives $D_{2, T}\left(Q^{1}, Q^{2}\right)=0$. With a Picard iteration of the type $Q^{n}=\Gamma\left(Q^{n-1}\right)$ and with the above arguments, we obtain that 


$$
\begin{aligned}
& \mathbf{E}\left[\sup _{t \in[0, T]}\left(X_{t}^{n}-X_{t}^{n-1}\right)^{2}+\left(\lambda_{t}^{n}-\lambda_{t}^{n-1}\right)^{2}\right] \\
& \leq L \int_{0}^{T} \mathbf{E}\left[\sup _{t \in[0, s]}\left(X_{t}^{n}-X_{t}^{n-1}\right)^{2}+\left(\lambda_{t}^{n}-\lambda_{t}^{n-1}\right)^{2}\right] d s \\
& \quad+\alpha^{2} T \int_{0}^{T} D_{2, s}\left(Q^{n-1}, Q^{n-2}\right)^{2} d s,
\end{aligned}
$$

that gives $D_{2, T}\left(Q^{n}, Q^{n-1}\right)^{2} \leq \frac{\left(e^{L T} T \alpha^{2}\right)^{n}}{n !} \int_{0}^{T} D_{2, s}\left(Q^{1}, Q^{0}\right)^{2} d s$, i.e. $\left\{Q^{n}\right\}_{n \in \mathbb{N}}$ is a Cauchy sequence for $D_{2, T}$ and therefore for a weaker, but complete, metric on $\mathcal{M}^{2}\left(\mathbf{C}\left([0, T], \mathbb{R}^{2}\right)\right)$.

Proof (Proof of Theorem 4) By the exchangeability of the components and of the dynamics, it is well-known that the thesis is implied by $\mathbf{E}\left[D_{2, T}\left(\mu^{N}, \mu\right)\right] \longrightarrow 0$ as $N \rightarrow+\infty$, where $\mu^{N}=\frac{1}{N} \sum_{i=1}^{N} \delta_{\left(X^{N}, \lambda^{N}\right)}$ (see [21]). Therefore, we apply this approach to prove the theorem. Fix a filtered probability space $\left(\Omega, \mathcal{F},\left\{\mathcal{F}_{t}\right\}_{t \in[0, T]}, \mathbf{P}\right)$, for any $N \in \mathbb{N}$, take a $2 N$-dimensional Brownian motion $\left\{B_{t}\right\}_{t \in[0, T]}$ and consider the coupled processes given by

$$
\left\{\begin{array}{l}
d X_{t}^{N, i}=\frac{\beta}{2} g^{\prime}\left(\lambda_{t}^{N, i}\right) d t+\frac{\rho^{\prime}\left(X_{t}^{N, i}\right)}{2 \rho\left(X_{t}^{N, i}\right)} d t+d B_{t}^{1, i} \\
d \lambda_{t}^{N, i}=-\alpha \lambda_{t}^{N, i} d t+\frac{1}{N} \sum_{j=1}^{N}\left(\frac{\beta}{2} g^{\prime}\left(\lambda_{t}^{N, j}\right)+\frac{\rho^{\prime}\left(X_{t}^{N, j}\right)}{2 \rho\left(X_{t}^{N, j}\right)}\right) d t+D d B_{t}^{2, i}+\frac{1}{N} \sum_{j=1}^{N} d B_{t}^{1, j},
\end{array}\right.
$$

$i=1, \ldots, N$, and

$$
\left\{\begin{array}{l}
d \bar{X}_{t}^{N, i}=\frac{\beta}{2} g^{\prime}\left(\bar{\lambda}_{t}^{N, i}\right) d t+\frac{\rho^{\prime}\left(\bar{X}_{t}^{N, i}\right)}{2 \rho\left(\bar{X}_{t}^{N, i}\right)} d t+d B_{t}^{1, i} \\
d \bar{\lambda}_{t}^{N, i}=-\alpha \bar{\lambda}_{t}^{N, i} d t+\left\langle\mu_{t}(d x, d l), \frac{\beta}{2} g^{\prime}(l)+\frac{\rho^{\prime}(x)}{2 \rho(x)}\right\rangle d t+D d B_{t}^{2, i}
\end{array}\right.
$$

$i=1, \ldots, N$, where the initial conditions are $\left(X_{0}^{N, i}, \lambda_{0}^{N, i}\right)=\left(\bar{X}_{0}^{N, i}, \bar{\lambda}_{0}^{N, i}\right)$ a.s., $\mu_{0}^{\otimes N}$ distributed. Similarly to the proof of Theorem 3 , we write explicitly, for all $i=1, \ldots, N$

$$
\begin{aligned}
\lambda_{t}^{N, i}-\bar{\lambda}_{t}^{N, i}= & \int_{0}^{t} e^{\alpha(s-t)}\left\langle\mu_{s}^{N}(d x, d l)-\mu_{s}(d x, d l), \frac{\beta}{2} g^{\prime}(l)+\frac{\rho^{\prime}(x)}{2 \rho(x)}\right\rangle d s \\
& +\frac{1}{N} \sum_{j=1}^{N} \int_{0}^{t} e^{\alpha(s-t)} d B_{s}^{1, i} .
\end{aligned}
$$

However, from (28), we see that, a.s. it holds

$$
\begin{aligned}
\int_{0}^{t} e^{\alpha(s-t)}\left\langle\mu_{s}^{N}(d x, d l), \frac{\beta}{2} g^{\prime}(l)+\frac{\rho^{\prime}(x)}{2 \rho(x)}\right\rangle d s= & \int_{0}^{t} e^{\alpha(s-t)} d\left\langle\mu_{s}^{N}(d x, d l), x\right\rangle \\
& -\frac{1}{N} \sum_{j=1}^{N} \int_{0}^{t} e^{\alpha(s-t)} d B_{s}^{1, i}
\end{aligned}
$$


and, consequently, we write

$$
\begin{aligned}
\lambda_{t}^{N, i}-\bar{\lambda}_{t}^{N, i}= & \frac{1}{N} \sum_{j=1}^{N} X_{t}^{N, j}-\mathbf{E}\left[\bar{X}_{t}^{N, i}\right]-e^{-\alpha t}\left(\frac{1}{N} \sum_{j=1}^{N} X_{0}^{N, j}-\mathbf{E}\left[\bar{X}_{0}^{N, i}\right]\right) \\
& -\alpha \int_{0}^{t}\left(\frac{1}{N} \sum_{j=1}^{N} X_{s}^{N, j}-\mathbf{E}\left[\bar{X}_{s}^{N, i}\right]\right) e^{-\alpha(t-s)} d s .
\end{aligned}
$$

Now let $\bar{\mu}^{N}=\frac{1}{N} \sum_{j=1}^{N} \delta_{\left(\bar{X}^{N, j}, \bar{\lambda}^{N, j}\right)}$; it is easy to see that, as in the proof of Theorem 3 , it holds

$$
\begin{aligned}
\mathbf{E}\left[D_{2, T}\left(\mu^{N}, \bar{\mu}^{N}\right)^{2}\right] \leq & \mathbf{E}\left[\sup _{t \in[0, T]}\left(X_{t}^{N, 1}-\bar{X}_{t}^{N, 1}\right)^{2}+\left(\lambda_{t}^{N, 1}-\bar{\lambda}_{t}^{N, 1}\right)^{2}\right] \\
\leq & L \int_{0}^{T} \mathbf{E}\left[\sup _{t \in[0, s]}\left(X_{t}^{N, 1}-\bar{X}_{t}^{N, 1}\right)^{2}+\left(\lambda_{t}^{N, 1}-\bar{\lambda}_{t}^{N, 1}\right)^{2}\right] d s \\
& +\alpha^{2} T \int_{0}^{T} \mathbf{E}\left[D_{2, s}\left(\mu^{N}, \mu\right)^{2}\right] d s,
\end{aligned}
$$

which, by an application of Gronwall's Lemma, implies that there exists $C_{T}>0$ such that

$$
\mathbf{E}\left[D_{2, T}\left(\mu^{N}, \bar{\mu}^{N}\right)^{2}\right] \leq C_{T} \int_{0}^{T} \mathbf{E}\left[D_{2, s}\left(\mu^{N}, \mu\right)^{2}\right] d s .
$$

Moreover, it is well known that $\mathbf{E}\left[D_{2, T}\left(\bar{\mu}^{N}, \mu\right)\right] \leq \beta(N)$ for some sequence $\beta(N)$ such that $\lim _{N \rightarrow \infty} \beta(N)=0$. Then, using (29), we have

$$
\begin{aligned}
\mathbf{E}\left[D_{2, T}\left(\mu^{N}, \mu\right)^{2}\right] & \leq \mathbf{E}\left[D_{2, T}\left(\mu^{N}, \bar{\mu}^{N}\right)^{2}\right]+\mathbf{E}\left[D_{2, T}\left(\bar{\mu}^{N}, \mu\right)^{2}\right] \\
& \leq C_{T} \int_{0}^{T} \mathbf{E}\left[D_{2, s}\left(\mu^{N}, \mu\right)^{2}\right] d s+\beta(N) \leq K_{T} \beta(N)
\end{aligned}
$$

for some $K_{T}>0$, which concludes the proof.

Acknowledgements The authors wish to thank Paolo Dai Pra for having suggested the idea of this work and for all the useful discussions on it and Marco Formentin for the suggestions and comments. This work was partially supported by the INdAM-GNAMPA Project 2017 "Collective periodic behavior in interacting particle systems". L. A. acknowledges the partial support by Centro Studi Levi Cases (Università di Padova).

Funding Funding were provided by Università degli Studi di Padova and Weierstrass Institute.

\section{References}

1. Acebrón, J.A., Bonilla, L.L., Vicente, C.J.P., Ritort, F., Spigler, R.: The Kuramoto model: a simple paradigm for synchronization phenomena. Rev. Mod. Phys. 77(1), 137 (2005)

2. Bertini, L., Giacomin, G., Pakdaman, K.: Dynamical aspects of mean field plane rotators and the Kuramoto model. J. Stat. Phys. 138(1-3), 270-290 (2010)

3. Carletti, T., Villari, G.: A note on existence and uniqueness of limit cycles for Liénard systems. J. Math. Anal. Appl. 307(2), 763-773 (2005)

4. Chen, W.-C.: Nonlinear dynamics and chaos in a fractional-order financial system. Chaos Solitons Fractals 36(5), 1305-1314 (2008)

5. Chen, X., Llibre, J., Zhang, Z.: Sufficient conditions for the existence of at least $n$ or exactly $n$ limit cycles for the Liénard differential systems. J. Differ. Equ. 242, 11-23 (2007) 
6. Collet, F., Dai Pra, P.: The role of disorder in the dynamics of critical fluctuations of mean field models. Electron. J. Probab 17(26), 1-40 (2012)

7. Collet, F., Dai Pra, P., Formentin, M.: Collective periodicity in mean-field models of cooperative behavior. Nonlinear Differ. Equ. Appl. NoDEA 22(5), 1461-1482 (2015)

8. Collet, F., Formentin, M., Tovazzi, D.: Rhythmic behavior in a two-population mean-field Ising model. Phys. Rev. E 94(4), 042139 (2016)

9. Dai Pra, P., Fischer, M., Regoli, D., Regoli, D.: A Curie-Weiss model with dissipation. J. Stat. Phys. 152(1), 37-53 (2013)

10. Dai Pra, P., Giacomin, G., Regoli, D.: Noise-induced periodicity: some stochastic models for complex biological systems. In: Mathematical Models and Methods for Planet Earth, pp. 25-35. Springer, Berlin (2014)

11. Dawson, D.A.: Critical dynamics and fluctuations for a mean-field model of cooperative behavior. J. Stat. Phys. 31(1), 29-85 (1983)

12. Ditlevsen, S., Löcherbach, E.: Multi-class oscillating systems of interacting neurons. Stoch. Process. Appl. 127, 1840-1869 (2016)

13. Eisele, T., Ellis, R.S.: Multiple phase transitions in the generalized Curie-Weiss model. J. Stat. Phys. 52(1-2), 161-202 (1988)

14. Ellis, R.S., Newman, C.M.: Limit theorems for sums of dependent random variables occurring in statistical mechanics. Zeitschrift für Wahrscheinlichkeitstheorie und verwandte Gebiete 44(2), 117-139 (1978)

15. Ermentrout, G.B., Terman, D.H.: Mathematical Foundations of Neuroscience, vol. 35. Springer, Berlin (2010)

16. Giacomin, G., Poquet, C.: Noise, interaction, nonlinear dynamics and the origin of rhythmic behaviors. Braz. J. Probab. Stat. 29(2), 460-493 (2015)

17. Ikeda, N., Watanabe, S.: Stochastic Differential Equations and Diffusion Processes. Elsevier, New York (2014)

18. Lindner, B., Garcia-Ojalvo, J., Neiman, A., Schimansky-Geier, L.: Effects of noise in excitable systems. Phys. Rep. 392(6), 321-424 (2004)

19. Odani, K.: Existence of exactly $N$ periodic solutions for Liénard systems. Funkcialaj Ekvacioj 39, $217-$ 234 (1996)

20. Scheutzow, M.: Noise can create periodic behavior and stabilize nonlinear diffusions. Stoch. Process. Appl. 20(2), 323-331 (1985)

21. Sznitman, A.-S.: Topics in propagation of chaos. In: Ecole d'Eté de Probabilités de Saint-Flour XIX1989, pp. 165-251. Springer, Berlin (1991)

22. Touboul, J.: The hipster effect: when anticonformists all look the same. arXiv preprint arXiv:1410.8001 (2014)

23. Touboul, J., Hermann, G., Faugeras, O.: Noise-induced behaviors in neural mean field dynamics. SIAM J. Appl. Dyn. Syst. 11(1), 49-81 (2012)

24. Turchin, P., Taylor, A.D.: Complex dynamics in ecological time series. Ecology 73(1), 289-305 (1992)

25. Weidlich, W., Haag, G.: Concepts and Models of a Quantitative Sociology: The Dynamics of Interacting Populations, vol. 14. Springer, Berlin (2012) 